\title{
Simple Hadronic Cascade Simulations
}

\author{
Fernando Sepúlveda and Claudio Dib \\ Dept. of Physics, Universidad Técnica Federico Santa María, Valparaíso, Chile
}

Received on 20 December, 2006

\begin{abstract}
We obtain results for the average number of muons at sea level in a proton-initiated vertical atmospheric cascade using a simple model of hadronic interactions based on the Hillas splitting algorithm. We study the muon yield at sea level as a function of the proton primary energy, varying the parameters of the interaction model in order to see the behavior of our results. We find that our results are in agreement with experimental data and with those of more sophisticated simulation models for some particular values of the model parameters.
\end{abstract}

Keywords: Hadronic cascade; Hillas splitting; Muon yield

\section{INTRODUCTION}

The hadronic component of an air shower is the one that continuously feeds the electromagnetic and muonic components through the decay of neutral and charged mesons respectively. The muon yield at detector level is a key signal to determine the energy and composition of the primary cosmic rays [1] and that is the motivation of this study.

In the simulation of hadronic cascades, the most important ingredient is, of course, the hadronic interaction model, several of which have been developed and are currently used in extensive air shower simulations [2, 3]. We simulate the longitudinal development of proton-initiated vertical cascades within a simple hadronic interaction model based on the Hillas splitting algorithm (HSA) which is computationally very fast [4]. According to this model only pions are produced as secondaries; their energies are generated as we explain in section II. Muons, on the other hand, arise from the decay of charged pions. This work is focused on studying the number of muons at sea level as a function of the proton primary energy. Varying the parameters of the interaction model allows us to see the behavior of the muon yield and we can select the values of the parameters for which our results best agree with known ones, either from experiments or other simulations.

In the next section the interaction model is explained and the average multiplicity in single interactions is studied. In sections III and IV we briefly discuss pion decay and the atmosphere model used. Section V is devoted to the cascade simulation and in section VI results regarding the muon content of the shower are discussed.

\section{HADRONIC INTERACTION MODEL}

As mentioned above, we study the development of the hadronic component of the shower using a HSA type model for the generation of secondaries in an inelastic hadronic ( $p$ Air or $\pi^{ \pm}$-Air) interaction. Here the secondaries are all pions and each kind is produced with a probability of one third. Hence, the interactions we consider in the simulations are of the following kind:

$$
\mathrm{p}\left(\pi^{ \pm}\right)+\operatorname{Air} \rightarrow \mathrm{p}\left(\pi^{ \pm}\right)+\operatorname{Air}+\mathrm{X},
$$

where $X$ represents any number of $\pi^{ \pm}$and $\pi^{0}$.
The energies of the secondaries at each interaction, whether the incoming particle is a proton or a charged pion, are generated by the HSA as follows:

1. Split the available energy of the incoming particle in two parts $A$ and $B$ at random.

2. Assign $A$ as the kinetic energy of the leading particle. This particle is of the same kind as the incoming one.

3. Split $B$ at random in $J=2^{N}$ branches ( $N$ a fixed positive integer).

4. Split one of the $J$ branches at random in two parts $A^{\prime}$ and $B^{\prime}$.

5. Assign $A^{\prime}$ as the kinetic energy of a pion.

6. From $B^{\prime}$ subtract $m_{\pi}$, split the rest at random in two parts and assign one piece as the kinetic energy of a pion. Call the other piece $B^{\prime}$ again.

7. Repeat step 6 until the energy remaining $\left(B^{\prime}\right)$ is less than some preassigned threshold $E_{t h}$. This value must be at least as large as $m_{\pi}$.

8. Once $E_{t h}$ has been reached, go back to step 4 with the next branch. Continue until all branches have been used.

This is an energy priority algorithm, which means that energy is conserved at each step, while the multiplicity is a result of the splitting process.

From the description of the algorithm given above, it is clear that the average multiplicity will increase with incoming energy. The algorithm is sensitive to the number of branches $J$. Tests were run for single interactions with $E_{t h}=m_{\pi}$ and $J=1,2,4$ and 8 in order to see how the average multiplicity varies as a function of the incoming energy. These results are shown in Fig. 1.

Observing Fig. 1 we can see that, for a fixed incoming energy, the average multiplicity grows with $J$. This occurs because if the threshold is reached in the splitting process (step 7 in the description above) when $J=1$, pion production ceases altogether. On the other hand, if the threshold is reached for one of the branches when $J>1$, pion production stops in that particular branch, but we have more branches where pions are created from. Hence the average multiplicity is higher. Since muons come from the decay of charged pions, we expect the muon number at sea level to increase notoriously with 


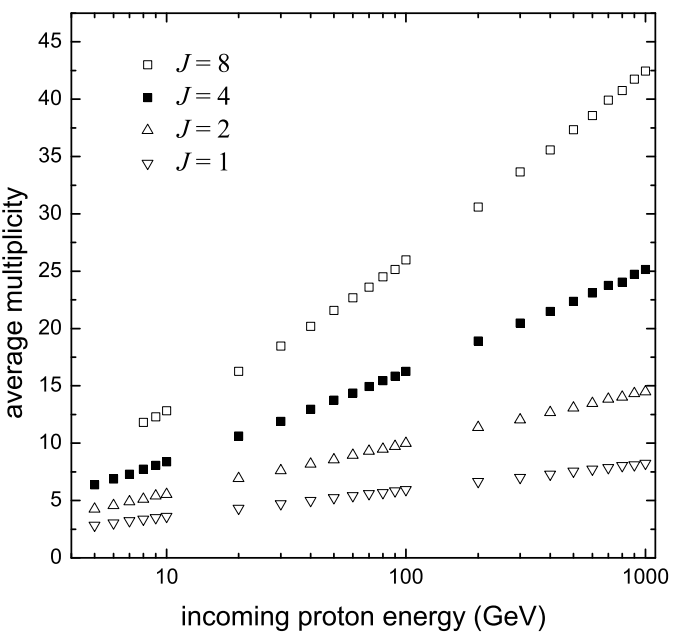

FIG. 1: Average number of secondaries in a p-Air interaction. Each point is the average multiplicity in ten thousand interactions.

growing $J$. The value $J=4$ is of special interest because it is suggested as a good interpolation between two more complex hadronic interaction models [3], GEISHA and QGSJET, in the energy region where neither of these models apply: GEISHA is valid for energies below a few tens of GeV and QGSJET is valid above several hundreds of $\mathrm{GeV}$.

\section{PION DECAY}

The lifetime of the charged pions is of order $10^{-8} \mathrm{~s}$, which makes the decay and interaction processes compete in the development of the shower. Hence the simulation must include both processes. The decay channels considered are:

$$
\pi^{ \pm} \rightarrow \mu^{ \pm}+v_{\mu}\left(\bar{v}_{\mu}\right)
$$

Since the secondary particles are isotropic in the CM, the energies of the products have flat distributions in their kinematic ranges [5]:

$$
\begin{array}{r}
\frac{m_{\mu}^{2}}{m_{\pi}^{2}} \cdot E_{\pi} \leq E_{\mu} \leq E_{\pi}, \\
0 \leq E_{\mathrm{v}} \leq E_{\pi} \cdot\left(1-\frac{m_{\mu}^{2}}{m_{\pi}^{2}}\right) .
\end{array}
$$

That is, the muon takes an energy with probability distributed uniformly within the ranges given in equation (2) and the neutrino carries the rest. These kinematic ranges are valid in the approximation of a highly relativistic pion and massless neutrinos. In our simulation only muons are tracked down, not the neutrinos.

The lifetime of the neutral pions is of order $10^{-16} \mathrm{~s}$, so even at the highest energies they decay almost instantaneously.
Their decay mode is:

$$
\pi^{0} \rightarrow 2 \gamma
$$

Since the photons are isotropic in the CM, their energies have a flat distribution in the range $\left(0, E_{\pi}\right)$ for highly relativistic pions.

\section{ISOTHERMAL ATMOSPHERE}

Since the natural units for interaction and decay paths are $\mathrm{g} / \mathrm{cm}^{2}$ and $\mathrm{km}$ respectively, we must have a model for the atmosphere in order to transform among these units. In the simulations we consider an isothermal atmosphere. Within this simple model, the ratio between vertical penetration depth (in $\mathrm{g} / \mathrm{cm}^{2}$ ) and density is constant, this constant represents a height scale and is defined as $h_{0}$ :

$$
h_{0} \equiv \frac{X}{\rho}=\frac{X}{-d X / d h} \propto \mathrm{T} .
$$

In this case, we have an exponential relation between vertical penetration depth and altitude [5]:

$$
X=1030 \cdot \exp [-h / 6.4] \mathrm{g} / \mathrm{cm}^{2},
$$

with $h$ in $\mathrm{km}$. This last relation, and its inverse, are used in the simulations to convert the units in pion decay distances from $\mathrm{km}$ to $\mathrm{g} / \mathrm{cm}^{2}$.

\section{SIMULATION OF THE CASCADES}

In this section we discuss in detail the method used for the simulation of the cascades. As was mentioned in section I, we simulate the longitudinal development of vertical cascades initiated by a high energy proton. The processes considered are the following: for protons we only consider inelastic interactions with atmospheric nuclei; for charged pions we consider interactions and decays; finally, for neutral pions only decays are taken into account. All distances are measured in $\mathrm{g} / \mathrm{cm}^{2}$. The basic idea of the algorithm can be summarized as follows:

1. For each particle with energy above the threshold of interest and altitude above sea level, determine the distance traveled until a decay or an interaction occurs, depending on the case at hand.

2. At that point, generate the corresponding secondaries. Record the depth at which each particle is created along with its energy and kind.

3. Go back to step one for each particle. 


\section{A. Protons in the Cascade}

In the case of protons we generate the distance traveled between interactions from an exponential probability distribution using the inversion method. We use an energy-dependent interaction length for protons (in $\mathrm{g} / \mathrm{cm}^{2}$ ) as suggested in ref. [6]:

$$
\lambda_{p}= \begin{cases}87 & \text { if } E \leq 0.1 \mathrm{TeV} \\ 80.8-2.78 \ln \left(\frac{E}{1 \mathrm{TeV}}\right) & \text { if } E>0.1 \mathrm{TeV},\end{cases}
$$

where $E$ is the energy of the incoming proton. Once the interaction point is known, the secondaries are generated using the HSA as described in section II. When the energy of the proton falls below $10 \mathrm{GeV}$, no more interactions are considered and the proton is only propagated until it reaches sea level.

\section{B. Charged Pions in the Cascade}

In the case of charged pions, we must consider both interaction and decay processes. This is done by sampling two distances, one for each process, and selecting the shortest one. If the selected process is interaction, the secondaries are generated at that point using the HSA as described in section II. Instead, if the selected process is decay, a muon and a neutrino are created using a flat distribution as discussed in section III. For interactions, the distance traveled is generated in the same way as for protons, but with an interaction length (in $\mathrm{g} / \mathrm{cm}^{2}$ ) [6]:

$$
\lambda_{\pi}= \begin{cases}116 & \text { if } E \leq 0.1 \mathrm{TeV} \\ 105-4.23 \ln \left(\frac{E}{1 \mathrm{TeV}}\right) & \text { if } E>0.1 \mathrm{TeV},\end{cases}
$$

where $E$ is the energy of the incoming pion. In an isothermal atmosphere, the decay length of charged pions, in $\mathrm{g} / \mathrm{cm}^{2}$, is given by [5]:

$$
d_{\pi}=\frac{E X}{\varepsilon_{\pi}}
$$

where $\varepsilon_{\pi}=115 \mathrm{GeV}, E$ is the energy and $X$ is the vertical penetration depth of the pion. Since $d_{\pi}$ depends on $X$, the distance traveled until decay is not so easily sampled. The natural units for the decay distance are kilometers. Hence, we must generate the decay distance in these units. This is achieved using the inversion method with an exponential probability distribution of mean path $\lambda_{d}=\gamma c \tau$, where $\gamma$ and $\tau$ are the lorentz factor and the mean life of the pion, respectively, together with unit conversion between $\mathrm{g} / \mathrm{cm}^{2}$ and $\mathrm{km}$ and viceversa using the exponential relation of equation (5).

The algorithm for the generation of the pion decay distance can be described as follows:

1. Take the initial position of the pion $X_{1}$, which is in units of $\mathrm{g} / \mathrm{cm}^{2}$, and transform it to a height $h_{1}$ in $\mathrm{km}$.

2. Calculate $\lambda_{d}=\gamma c \tau$ for the pion in question.

3. Generate the decay distance $l_{d}$ from an exponential distribution of mean path $\lambda_{d}$.
4. With the decay distance $l_{d}$, calculate the height $h_{2}$ at which the pion decays: $h_{2}=h_{1}-l_{d}$.

5. Transform $h_{2}$ to $\mathrm{g} / \mathrm{cm}^{2}$ to obtain the final position of the pion $X_{2}$.

6. The decay distance in $\mathrm{g} / \mathrm{cm}^{2}$ is thus $X_{2}-X_{1}$.

The probability for a pion to decay as it traverses a small $\Delta X$ of atmosphere is $\Delta X / d_{\pi}$. As can be seen from equation (8), this probability is negligible if $E \gg \varepsilon_{\pi}$. On the other hand, decay is the dominating process if $E \ll \varepsilon_{\pi}$. In the simulations we separate three energy regions for pions:

1. When $E>1 \mathrm{TeV}$ only interactions are considered. The distance traveled is generated from an exponential probability distribution with mean free path $\lambda_{\pi}$ and secondaries are generated using the HSA.

2. When $E<10 \mathrm{GeV}$ only decays are considered. The distance traveled until decay is generated as discussed above. The secondaries are created as discussed in section III.

3. When $10 \mathrm{GeV}<E<1 \mathrm{TeV}$, two distances are sampled, one for each process just like above, and the process corresponding to the shortest distance is the one that occurs.

\section{Neutral Pions in the Cascade}

In the case of neutral pions, due to their short lifetime, only decays are considered. The decay is simulated right where the neutral pion is created. The photons produced share the energy of the parent $\pi^{0}$ at random, but here we do not treat this part of the process, as it feeds into the electromagnetic component of the shower, which does not subsequently affect the hadronic development.

\section{Muons in the Cascade}

Muons above threshold are propagated from their point of creation down to sea level without considering any interaction or decay processes. Since we use thresholds above $10 \mathrm{GeV}$, disregarding decays does not affect the results.

\section{RESULTS AND CONCLUSIONS}

We concentrate on the muon content of the shower at sea level. As mentioned in section II the key parameter in the simulation is the number of branches $J$ in the HSA. We study the muon number at sea level as a function of primary energy and its behavior as $J$ is varied. The simulations were carried out for primary protons with energies between $10^{14}$ and $10^{16} \mathrm{eV}$ using $J=1,2,4$ and 8 for each primary energy. The results are shown in Fig. 2. 


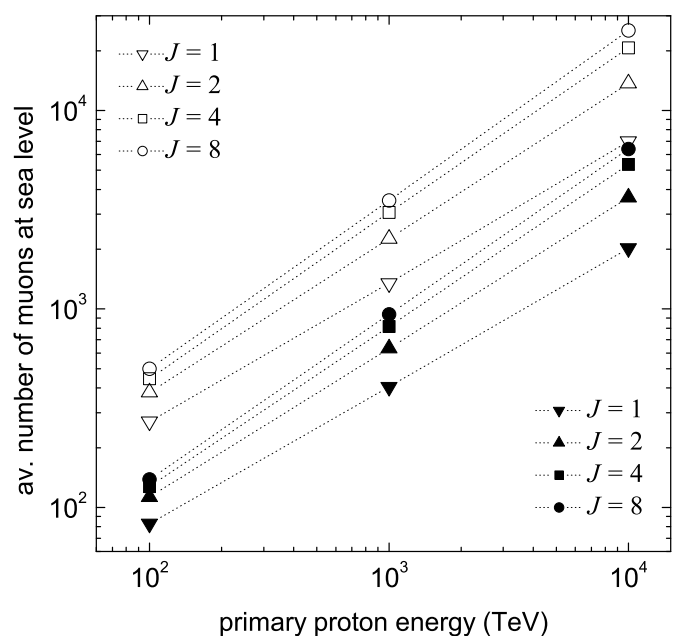

FIG. 2: Average number of muons at sea level as a function of primary energy. Empty and solid symbols correspond to muon energy thresholds of $10 \mathrm{GeV}$ and $30 \mathrm{GeV}$ respectively. Each point is obtained by averaging the muon content in one hundred vertical showers.

\begin{tabular}{|c|c|c|c|c|c|c|c|}
\hline$E_{t h}[\mathrm{GeV}]$ & $J$ & $C$ & $\beta$ & $E_{t h}[\mathrm{GeV}]$ & $J$ & $C$ & $\beta$ \\
\hline 10 & 1 & 10.442 & 0.7057 & 30 & 1 & 3.3695 & 0.6943 \\
\hline 10 & 2 & 10.509 & 0.7783 & 30 & 2 & 3.4755 & 0.7548 \\
\hline 10 & 4 & 9.6191 & 0.8335 & 30 & 4 & 3.0279 & 0.8113 \\
\hline 10 & 8 & 9.8028 & 0.8528 & 30 & 8 & 2.9945 & 0.8323 \\
\hline
\end{tabular}

TABLE I: Parameters in the power law for the average number of muons at sea level of equation (9).

Irrespective of the value of $J$ used, we find that the average number of muons with energies above 10 and $30 \mathrm{GeV}$ at sea level as a function of the primary energy is very well fitted by a power law:

$$
N_{\mu}=C \cdot E_{0}^{\beta},
$$

where all correlation coefficients satisfy $R^{2}>0.999$ and $0<\beta<1$. The values of $\beta$ and $C$ depend on the muon en- ergy threshold and on the particular $J$ used in the HSA. These values are shown in table I. The general result expressed in equation (9) is expected from what is known from more complex models and from experimental results $[2,5]$.

In table I we can see that for each value of $J$ the exponent $\beta$ is larger for the $10 \mathrm{GeV}$ threshold and grows smoothly as $J$ goes from 1 to 8 . As expected, Fig. 2 shows a marked increase in the number of muons at sea level as $J$ goes from 1 to 8 for every primary energy. However, each time $J$ is doubled this increase is progressively slower. For example, take the case of the $10 \mathrm{GeV}$ threshold and $E_{0}=100 \mathrm{TeV}$. When $J$ goes from 1 to $2, N_{\mu}$ grows in a factor of 1.4; when $J$ goes from 2 to $4, N_{\mu}$ grows in a factor of 1.17; and when $J$ goes from 4 to $8, N_{\mu}$ grows only in a factor of 1.12. This behavior occurs because as the average multiplicity in the HSA grows with $J$, the average energy per pion decreases, which means the muons produced in the decays are less energetic.

The values of $C$ and $\beta$ for $J=4$ and 8 are in very good agreement with those obtained by Stanev [2] with the SYBILL model of hadronic interactions [7] and by the simple, deterministic model of Matthews [8]. Furthermore, these results are in very good agreement with the experimental results reported by the Akeno group [9]. On the other hand; the results obtained with $J=1$ and 2 have values for $\beta$ that are too low, especially for $J=1$. This is due to the extremely low multiplicity given by the HSA for these values of $J$, as can be seen in Fig. 1.

This simple model for the simulation can be improved in several ways. We can consider a more realistic model for the atmosphere. Also, we can improve on the hadronic interaction model modifying the HSA algorithm by setting the number of branches $J$ to be energy-dependent and fit hadronic data more precisely.

It is natural to conclude that the parameters in equation (9) are sensitive to the hadronic interaction model. The advantage of this approach lies in the computational speed of the HSA and in its flexibility.

\section{Acknowledgments}

This study has been partially funded by Fondecyt, Chile grant no. 1030254 .
[1] A. Haungs, H. Rebel, and M. Roth, Rep. Prog. Phys. 66, 1145 (2003).

[2] Todor Stanev, High Energy Cosmic Rays, Springer-Praxis 2004.

[3] J. Knapp, D. Heck, S.J. Sciutto, M.T. Dova, and M. Risse, Astropart. Phys. 19, 77 (2003).

[4] A. Hillas, Proc. 17th Int. Cosm. Ray Conf. 8, 193 (1981).

[5] T. K. Gaisser, Cosmic Rays and Particle Physics, Cambridge University press 1990.

[6] $\lambda$ is taken as constant for incoming $E<0.1 \mathrm{TeV}$, as suggested in ref. [5], and decreasing logarithmically for larger $E$. This loga- rithmic term is fitted to the values of Table 5.1 in ref. [5]. These values agree with the $p-p$ and $\pi-p$ collisions listed in S. Eidelman et al., Phys. Lett. B592, 1 (2004) and converted to $p$-Air and $\pi$-Air collisions, respectively, according to B. Kopeliovich et al., Phys. Rev. D 39, 769 (1989).

[7] R.S. Fletcher, T.K. Gaisser, P. Lipari, and T. Stanev, Phys. Rev. D 50, 5710 (1994).

[8] J. Matthews, Proc. 27th Int. Cosm. Ray Conf. 1, 261 (2001). [9] M. Nagano et al., J. Phys. G 10, 1295 (1984). 